\title{
EFFECT OF HF ACID ETCHING DURATION ON SURFACE ROUGHNESS OF NEWLY INTRODUCED GLASS CERAMIC MATERIAL
}

\author{
Noha Mohamed Abdel Zziz Murad*
}

\begin{abstract}
Purpose: The aim of this in-vitro study will be directed to evaluating the effect of different hydrofluoric acid etching durations on the surface roughness of lithium silicate based glass ceramics obsidian and e-max, microstructure changes also color difference.
\end{abstract}

Materials and methods: Eighty disc samples were grouped according to material type into two groups; obsidian group $(n=40)$ and e-max group $(n=40)$, each group was subdivided into four groups according the duration of hydrofluoric acid (HF) application into: Subgroup I (control) (n=10) without surface treatment. Subgroup II, (n=10) 20 seconds Subgroup III, (n=10): 40 seconds. Subgroup IV, $(\mathrm{n}=10)$ : 60 seconds. All surface treated samples will be subjected to: Surface roughness examination, Surface topographic analysis using SEM and profilometer, and color difference

Results: the surface roughness test revealed that; the highest mean value was recorded in 60 second etch, followed by $40 \mathrm{sec}$ etch then 20 second etch; with the least value recorded in control (no etch). ANOVA test revealed that the difference between etching subgroups was statistically significant $(\mathrm{p}<0.0001)$. Also higher mean value was recorded in E-max with statistically significant difference $(\mathrm{p}=0.014)$. Effect of etching time on color the highest mean value was recorded in 60 second etch, followed by $40 \mathrm{sec}$ etch then 20 second etch; with the least value recorded in control (no etch). ANOVA test revealed that the difference between etching time was statistically significant $(\mathrm{p}<0.0001)$.Also a higher mean value was recorded in E-max with statistically significant difference $(\mathrm{p}<0.0001)$,

Conclusion E-max group showed higher means of surface roughness test and color difference rather than obsidian. Also 60 second etch showed the highest mean values of surface roughness test and color difference followed by 40 second etch followed by 40 second etch.

\footnotetext{
* DDS In Fixed Prosthodontics Must University
} 


\section{INTRODUCTION}

Esthetic aspects of dental treatment are becoming increasingly important to patients, thus increasing the demand for all ceramic restorations. Since the introduction of reinforced feldspathic Porcelain in $1965^{(1)}$, new materials and processing technology for all ceramic restorations with significantly improved physical and mechanical properties are available at present ${ }^{(2,3)}$.

One of the early generations of glass ceramics was Leucite-based glass ceramics IPS Empress I (Ivoclar Vivadent) ${ }^{(4,5)}$. Its major advantage over other types of ceramic materials is a translucency similar to that of enamel, providing a "chameleon effect" that permits light to be reflected, scattered, and absorbed throughout the restoration and the underlying tooth structure and allows the restoration to blend with the natural tooth ${ }^{(6,7)}$. Despite its limited in use to single unit complete-coverage restorations in the anterior segment due to its low mechanical properties ${ }^{(8)}$.

In order to enable glass-ceramics to be used in the fabrication of dental bridges and single crowns in the molar region, the strength and toughness of these materials had to be increased. A significant increase in these parameters was achieved by introducing the Lithium disilicate glassceramics $(9,10)$.

The next generation of lithium disilicates (IPS e.max Press IPS e.max CAD, Ivoclar Vivadent, Amherst, NY, USA) ${ }^{(11,12)}$. Is an innovative allceramic system that comprises lithium disilicate (LS2) glass ceramic for the press and CAD/CAM technologies ${ }^{(13)}$. Restorations can be fabricated from this type of resistant glass ceramic with either lost-wax hot pressing techniques or modern $\mathrm{CAD} /$ CAD milling procedures ${ }^{(14,15)}$.

Recently in the past few years a new glass ceramic -lithiumsilicate based-wasintroducedunder thename of Obsidian. Obsidian is a monolithic restoration. It may use as CAD/ CAM milling blocks or pressed to metal results in a restoration with superior strength. Obsidian blocks are lithium silicate glass ceramic that contain 20 elemental oxides including Zirconia. Additionally, the Obsidian milling block owes its excellent properties due to a very high content of ultra-nanometer-size lithium silicate and lithium phosphate crystals. Obsidian milling block is supplied in a partially crystallized phase, which is to be milled using CEREC MC XL milling machine. Obsidian is highly esthetic and chip resistant. It exhibits excellent translucency, resulting in superior esthetics. It is indicated for crowns, inlays, onlays, and veneers; possesses above average flexural strength and is recommended mainly for anterior and premolar crowns ${ }^{(16)}$.

An important aspect required for the success of such restorations is the establishment of proper adhesion between substrate and adherent ${ }^{(17)}$. In this sense, the gold-standard protocol for resin bonding to glass ceramics is the etching with hydrofluoric acid (HF) followed by the application of a silane coupling agent (chemical and micro-mechanical bond) ${ }^{(18,19)}$.

Variations in HF acid etching (time and concentration) have been shown to change the surface micro-morphology of glass ceramics (Traini et al., 2016) ${ }^{(20)}$ and resin adhesion (Leite et al., 2013; Venturini et al., 2015) ${ }^{(21,22)}$, for ceramic surface treatment, the acid reacts with the glass matrix that contains silica and forms hexafluorosilicates. This glass matrix is selectively removed and the crystalline structure is exposed. As a result, the surface of the ceramic becomes rough, which is expected for micromechanical retention on the ceramic surface. This roughly etched surface also helps to provide more surface energy prior to combining with the silane solution.

The study have reported positive effects of hydrofluoric acid (HF) etching on the strength of glasses by removing or stabilizing surface defects 
and on surface topography increasing roughness for adhesive bonding.

\section{MATERIALS AND METHODS}

Forty $(\mathrm{N}=40)$ samples for each material (lithium-silicate glass ceramic (Obsidian; Prismatik Dentalcraft, Glidewell Labs.) and (lithium-disilicate glass ceramic (e max CAD Ivoclar Vivadent, Schaan, Liechtenstein). will be constructed of 10.2 $\mathrm{mm}$ diameter and $1.3 \mathrm{~mm}$ thickness. The dimensions of the samples were confirmed using digital caliper.

The ceramic slices were wet polished using 1000-grit silicon carbide paper and polishing paste to remove external irregular scratches and defects. All ceramic specimens were sonically cleaned in distilled water for $15 \mathrm{~min}$.

Samples are divided into four groups according the duration of hydrofluoric acid (HF) application as follows:

1. Group I (control) $(\mathrm{n}=10)$ : as received samples without surface treatment.

2. Group II, $(n=10)$ : ceramic surfaces etched with HF for 20 seconds; following the manufacturer's recommendations.

3. Group III, $(n=10)$ : ceramic surfaces etched with HF for 40 seconds.

4. Group IV, $(n=10)$ : ceramic surfaces etched with HF for 60 seconds.

- All surface treated samples will be subjected to:

1. Surface roughness examination. Surface Roughness analysis was done using photographed using USB Digital microscope. With a builtin camera (Scope Capture Digital Microscope, Guangdong, and China) connected with an IBM compatible personal computer using a fixed magnification of 120X. The cropped images were analyzed using WSxM software. Calibration was made by comparing an object of known size (a ruler in this study) with a scale generated by the software.
- Surface topographic analysis using SEM and profilometer.

Using SEM Model Quanta 250 FEG (Field Emission Gun), with accelerating voltage 30 K.V. and magnification $14 x$ up to 1000000 . The magnification used in this study was $2000 x$.

\section{- Color stability testing $(\Delta \mathrm{E})$.}

Aspectrophotometer(FEICompany,Netherlands) was used for the reflectance measurements of the disc samples. The color change value $(\Delta \mathrm{E})$ was calculated by the following equation:

$$
\Delta \mathrm{E}=\left[\left(\Delta \mathrm{L}^{*}\right) 2+\left(\Delta \mathrm{a}^{*}\right) 2+\left(\Delta \mathrm{b}^{*}\right) 2\right] 1 / 2 .
$$

$\mathrm{L}^{*}$ stands for lightness, $\mathrm{a}^{*}$ for green-red and $\mathrm{b}^{*}$ for blue-yellow. $\Delta \mathrm{L}^{*}, \Delta \mathrm{a}^{*}, \Delta \mathrm{b}^{*}$ correspond to the differences between specimens after etching and after bonding

Obtained data will be tabulated and statistically analyzed.

\section{RESULTS}

\section{I-Effect of etching time on roughness}

\section{a) Comparison within the same group}

In E-max group, the highest mean value was recorded in 60 second etch, followed by $40 \mathrm{sec}$ etch then, control (no etch); with the least value recorded in 20 second etch. ANOVA test revealed that the difference between etching time was statistically significant $(\mathrm{p}<0.0001)$. Tukey's post hoc test revealed a significant difference between each 2 etching subgroups (table 1, Fig. 1)

In Obsidian group, the highest mean value was recorded in 40 second etch, followed by $60 \mathrm{sec}$ etch then, control (no etch); with the least value recorded in 20 second etch. ANOVA test revealed that the difference between etching subgroups was not statistically significant ( $\mathrm{p}=0.361$ ), (Table 1, Fig. 1) 
TABLE (1) Values of roughness and effect of etching time within the same group (ANOVA test)

\begin{tabular}{|c|c|c|c|c|c|c|c|c|c|c|}
\hline \multirow{2}{*}{\multicolumn{2}{|c|}{ Roughness }} & \multirow[b]{2}{*}{ Mean } & \multirow{2}{*}{$\begin{array}{l}\text { Std. } \\
\text { Dev }\end{array}$} & \multirow{2}{*}{$\begin{array}{l}\text { Std. } \\
\text { Error }\end{array}$} & \multicolumn{2}{|c|}{$\begin{array}{c}\text { 95\% Confidence Interval for } \\
\text { Mean }\end{array}$} & \multirow[b]{2}{*}{ Min } & \multirow[b]{2}{*}{ Max } & \multirow[t]{2}{*}{$\mathrm{F}$} & \multirow[t]{2}{*}{$\mathrm{P}$} \\
\hline & & & & & Lower Bound & Upper Bound & & & & \\
\hline \multirow[t]{4}{*}{ EMAX } & Control (No etch) & $.2545^{\mathrm{c}}$ & .00 & .00 & .25 & .26 & .25 & .26 & 108.002 & $.000 *$ \\
\hline & $20 \mathrm{sec}$. etch & $.2523^{\mathrm{d}}$ & .00 & .00 & .25 & .25 & .25 & .25 & & \\
\hline & $40 \mathrm{sec}$. etch & $.2567^{\mathrm{b}}$ & .00 & .00 & .26 & .26 & .26 & .26 & & \\
\hline & $60 \mathrm{sec} . \mathrm{etch}$ & $.2647^{\mathrm{a}}$ & .00 & .00 & .26 & .27 & .26 & .27 & & \\
\hline \multirow[t]{4}{*}{ Obsidian } & Control (No etch) & $.2538^{\mathrm{a}}$ & .00 & .00 & .25 & .26 & .24 & .26 & & \\
\hline & $20 \mathrm{sec}$. etch & $.2505^{\mathrm{a}}$ & .01 & .00 & .24 & .26 & .22 & .27 & 1.089 & $.361 \mathrm{NS}$ \\
\hline & $40 \mathrm{sec}$. etch & $.2567^{\mathrm{a}}$ & .00 & .00 & .26 & .26 & .25 & .26 & & \\
\hline & $60 \mathrm{sec}$. etch & $.2546^{\mathrm{a}}$ & .01 & .00 & .25 & .26 & .21 & .27 & & \\
\hline
\end{tabular}

Significance level P<0.05, *Significant, NS=non-significant

Tukey's post hoc test: Within the same comparison, means sharing the same superscript letter are not significantly different

TABLE (2) Comparison of values of roughness between E-max and Obsidian (independent $t$ test)

\begin{tabular}{|c|c|c|c|c|c|}
\hline Etching & Group & Mean & SD & $\mathrm{t}$ & $\mathrm{P}$ \\
\hline \multirow{2}{*}{ Control } & $E \max$ & .2545 & .00 & \multirow[t]{2}{*}{.663} & \multirow{2}{*}{$0.516 \mathrm{NS}$} \\
\hline & Obsidian & .2538 & .00 & & \\
\hline \multirow{2}{*}{$20 \mathrm{sec}$. etch } & $E \max$ & .2523 & .00 & \multirow[t]{2}{*}{.525} & \multirow{2}{*}{$0.608 \mathrm{NS}$} \\
\hline & Obsidian & .2505 & .01 & & \\
\hline \multirow{2}{*}{$40 \mathrm{sec}$. etch } & $\mathrm{E} \max$ & .2567 & .00 & \multirow[t]{2}{*}{0} & \multirow{2}{*}{$1 \mathrm{NS}$} \\
\hline & Obsidian & .2567 & .00 & & \\
\hline \multirow{2}{*}{$60 \mathrm{sec}$ etch } & $\mathrm{E} \max$ & .2647 & .00 & \multirow[t]{2}{*}{7.35} & \multirow{2}{*}{$0.00 *$} \\
\hline & Obsidian & .2546 & .01 & & \\
\hline
\end{tabular}

Significance level $P<0.05$, *Significant, NS=non-significant

TABLE (3a) Comparison of Values of roughness in different etch time (2 ways ANOVA)

\begin{tabular}{|c|c|c|c|c|c|c|}
\hline & & & \multicolumn{2}{|c}{$95 \%$ Confidence Interval } & \multirow{2}{*}{ F } & P \\
\cline { 4 - 6 } Etch time & Mean & Std. Error & Lower Bound & Upper Bound & & \\
\hline Control & $.254^{\mathrm{b}}$ & .001 & .252 & .257 & 9.560 & $.000^{*}$ \\
\hline $20 \mathrm{sec}$ etch & $.251^{\mathrm{c}}$ & .001 & .249 & .254 & & \\
\hline $40 \mathrm{sec}$ etch & $.256^{\mathrm{b}}$ & .001 & .253 & .258 & & \\
\hline $60 \mathrm{sec}$ etch & $.261^{\mathrm{a}}$ & .001 & .258 & .263 & & \\
\hline
\end{tabular}

Significance level $P<0.05$, *Significant

Tukey's post hoc test: means sharing the same superscript letter are not significantly different 


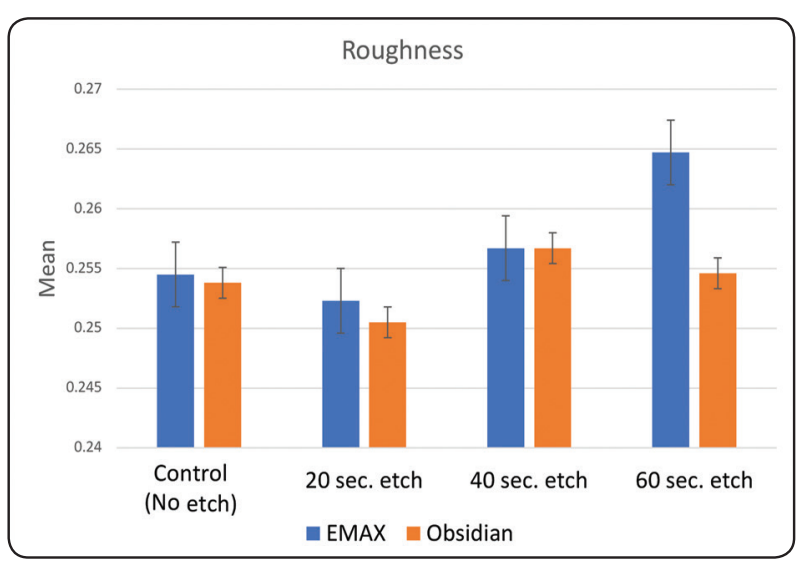

Fig. (1) Bar chart showing mean values of roughness and effect of etching time in both groups

\section{b) Comparison between groups}

In control subgroups, a higher mean value was recorded in E-max, with no significant difference $(\mathrm{p}=0.516)$, (Table 2, Fig. 1)

In $20 \mathrm{sec}$ etch subgroups, a higher mean value was recorded in E-max, with no significant difference ( $\mathrm{p}=0.608)$, (Table 2, Fig. 1)

In $40 \mathrm{sec}$ etch subgroups, the same mean value was recorded in both groups, with no statistically significant difference ( $\mathrm{p}=1)$, (Table 2, Fig. 1)
In $60 \mathrm{sec}$ etch subgroups, a higher mean value was recorded in E-max, with a significant difference $(\mathrm{p}=0.00)$, (Table 2, Fig. 1)

\section{C) Interaction of variables}

Two ways ANOVA test was used to study the interaction of both etching time and group variables. Regarding the etching time variable, the highest mean value was recorded in 60 second etch, followed by $40 \mathrm{sec}$ etch, then control (no etch); with the least value recorded in 20 second etch. Two ways ANOVA test revealed that the difference between etching time was statistically significant $(\mathrm{p}<0.0001)$. Tukey's post hoc test revealed a significant difference between each 2 etching times (Table 3a)

Regarding the group variable, a higher mean value was recorded in E-max with statistically significant difference $(\mathrm{p}=0.014)$, (Table $3 \mathrm{~b}$ )

Two ways ANOVA test revealed that the effect of interaction of both variables was not statistically significant $(\mathrm{p}=0.176)$, (Table $3 \mathrm{c})$

\section{II- Effect of etching time on color}

\begin{tabular}{|c|c|c|c|c|c|c|}
\hline \multirow{2}{*}{ Group } & \multirow{2}{*}{ Mean } & Std. Error & \multicolumn{2}{|c|}{$95 \%$ Confidence Interval } & \multirow{2}{*}{ F } & P \\
\cline { 4 - 5 } & & Lower Bound & Upper Bound & & \\
\hline E max & .257 & .001 & .255 & .259 & 6.247 & $.014^{*}$ \\
\hline Obsidian & .254 & .001 & .252 & .256 & & \\
\hline
\end{tabular}

Significance level $\mathrm{P}<0.05$, *Significant

TABLE (3c) Results of 2 ways ANOVA test for roughness

\begin{tabular}{|c|c|c|c|c|c|}
\hline Source & \multicolumn{1}{c}{ Type III Sum of Squares } & $\mathrm{df}$ & \multicolumn{2}{c|}{ Mean Square } & \multicolumn{1}{c|}{ F } \\
\hline -Etch_time & .001 & 3 & .000 & 9.560 & $.000^{*}$ \\
-Group & .000 & 1 & .000 & 6.247 & $.014^{*}$ \\
Etch time* Group & .000 & 3 & .000 & 1.679 & $.176 \mathrm{NS}$ \\
\hline
\end{tabular}

Significance level $\mathrm{P}<0.05$, *Significant, NS=non-significant 


\section{a-Comparison within the same group}

In E-max group, the highest mean value was recorded in 60 second etch, followed by $40 \mathrm{sec}$ etch then 20 second etch; with the least value recorded in control (no etch). ANOVA test revealed that the difference between etching time was statistically significant $(\mathrm{p}<0.0001)$. Tukey's post hoc test revealed a significant difference between each 2 etching subgroups (Table 4, Fig. 2)

In Obsidian group, the highest mean value was recorded in 60 second etch, followed by 40 sec etch then 20 second etch; with the least value recorded in control (no etch). ANOVA test revealed that the difference between etching subgroups was statistically significant $(\mathrm{p}<0.0001)$. Tukey's post hoc test revealed a significant difference between each 2 etching subgroups (Table 4, Fig. 2)

\section{b-Comparison between groups}

In control subgroups, a higher mean value was recorded in E-max group, with statistically significant difference $(\mathrm{p}<0.0001), \quad($ Table 5, Fig. 2)

In $20 \mathrm{sec}$ etch subgroups, a higher mean value was recorded in E-max, with statistically significant difference ( $<<0.0001$ ), (Table 5, Fig. 2)
In $40 \mathrm{sec}$ etch subgroups, a higher mean value was recorded in E-max, with statistically significant difference ( $<<0.0001)$, (Table 5, Fig. 2)

In $60 \mathrm{sec}$ etch subgroups, a higher mean value was recorded in E-max, with statistically significant difference ( $<<0.0001$ ), (Table 5, Fig. 2)

\section{C-Interaction of variables}

Two ways ANOVA test was used to study the interaction of both etching time and group variables. Regarding the etching time variable, the highest mean value was recorded in 60 second etch, followed by $40 \mathrm{sec}$ etch, then 20 second etch; with the least value recorded in control (no etch). Two ways ANOVA test revealed that the difference between etching time was statistically significant $(\mathrm{p}<0.0001)$. Tukey's post hoc test revealed a significant difference between each 2 etching times (Table 6a)

Regarding the group variable, a higher mean value was recorded in E-max with statistically significant difference $(\mathrm{p}<0.0001)$, (Table $6 \mathrm{~b}$ )

Two ways ANOVA test revealed that the effect of interaction of both variables was statistically significant $(p<0.0001)$, (Table 6c)

TABLE (4) Values of colour difference and effect of etching time within the same group (ANOVA test)

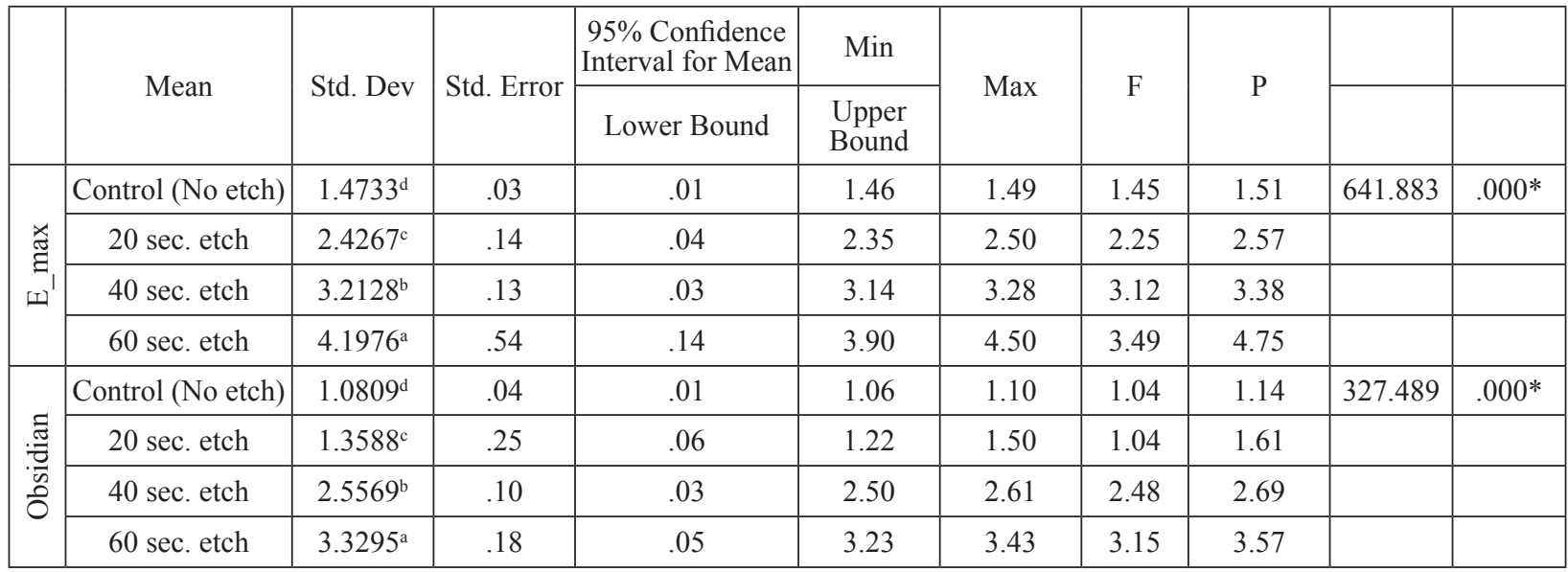

Significance level $P<0.05$, *Significant

Tukey's post hoc test: Within the same comparison, means sharing the same superscript letter are not significantly different 
TABLE (5) Comparison of values of color difference between E-max and Obsidian (independent $t$ test)

\begin{tabular}{|c|c|c|c|c|c|}
\hline Etching & Group & Mean & SD & $\mathrm{t}$ & $\mathrm{P}$ \\
\hline \multirow{2}{*}{ Control } & $E \max$ & 1.4733 & .03 & \multirow{2}{*}{-30.934} & \multirow{2}{*}{$0.00^{*}$} \\
\hline & Obsidian & 1.0809 & .04 & & \\
\hline \multirow{2}{*}{$20 \mathrm{sec}$. etch } & $E \max$ & 2.4267 & .14 & \multirow{2}{*}{-14.632} & \multirow{2}{*}{$0.00^{*}$} \\
\hline & Obsidian & 1.3588 & .25 & & \\
\hline \multirow{2}{*}{$40 \mathrm{sec}$. etch } & $\mathrm{E} \max$ & 3.2128 & .13 & \multirow{2}{*}{5.632} & \multirow{2}{*}{$0.00^{*}$} \\
\hline & Obsidian & 2.5569 & .10 & & \\
\hline \multirow{2}{*}{$60 \mathrm{sec}$ etch } & $\mathrm{E} \max$ & 4.1976 & .54 & \multirow{2}{*}{6.61} & \multirow{2}{*}{$0.00^{*}$} \\
\hline & Obsidian & 3.3295 & .18 & & \\
\hline
\end{tabular}

Significance level P<0.05, *Significant

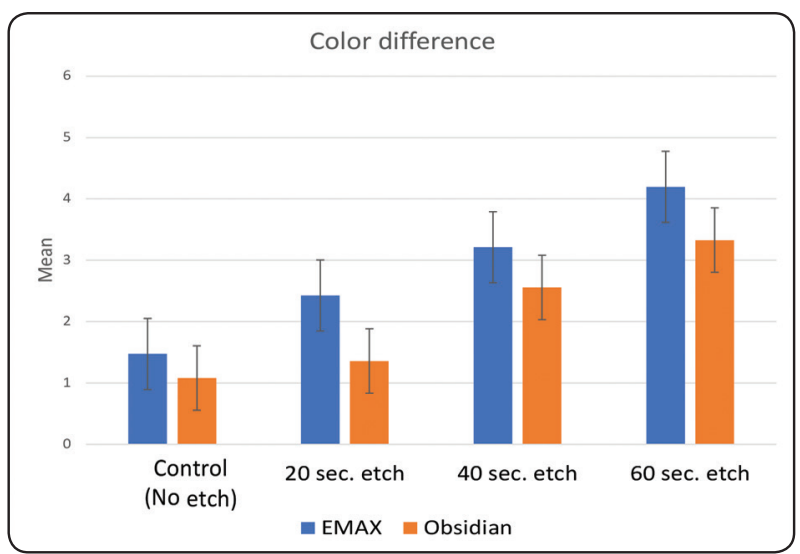

Fig. (2) Bar chart showing mean values of color difference and effect of etching time in both groups

\section{Correlation between roughness and color (Table 7, Fig. 3)}

Pearson correlation test revealed a statistically significant strong positive correlation between color difference and roughness in E-max group $(\mathrm{p}<0.0001)$

Pearson correlation test revealed a nonsignificant weak positive correlation between color difference and roughness in Obsidian group $(\mathrm{p}=0.446)$

Pearson correlation test revealed a statistically significant moderate positive correlation between color difference and roughness in both groups (all together $)(\mathrm{p}<0.0001)$

TABLE (6A) Comparison of Values of color difference in different etch time (2 ways ANOVA)

\begin{tabular}{|c|c|c|c|c|c|c|}
\hline \multirow{2}{*}{ Etch_time } & \multirow{2}{*}{ Mean } & \multirow{2}{*}{ Std. Error } & \multicolumn{2}{|c|}{$95 \%$ Confidence Interval } & \multirow{2}{*}{ F } & \multirow{2}{*}{ P } \\
\cline { 4 - 6 } & & & Lower Bound & Upper Bound & & \\
\hline Control & $1.277^{\mathrm{d}}$ & .043 & 1.193 & 1.362 & 640.808 & \multirow{2}{*}{$.000^{*}$} \\
\hline $20 \mathrm{sec}$ etch & $1.893^{\mathrm{c}}$ & .043 & 1.808 & 1.977 & & \\
\hline $40 \mathrm{sec}$ etch & $2.943^{\mathrm{b}}$ & .043 & 2.859 & 3.028 & & \\
\hline $60 \mathrm{sec}$ etch & $3.705^{\mathrm{a}}$ & .043 & 3.621 & 3.790 & & \\
\hline
\end{tabular}

Significance level P<0.05, *Significant

Tukey's post hoc test: means sharing the same superscript letter are not significantly different 
TABLE (6B) Comparison of Values of color difference in different groups (2 ways ANOVA)

\begin{tabular}{|c|c|c|c|c|c|c|}
\hline \multirow{2}{*}{ Group } & \multirow{2}{*}{ Mean } & \multirow{2}{*}{ Std. Error } & \multicolumn{2}{|c|}{$95 \%$ Confidence Interval } & \multirow{2}{*}{$\mathrm{F}$} & \multirow{2}{*}{$\mathrm{P}$} \\
\cline { 4 - 5 } & & & Lower Bound & Upper Bound & & \\
\hline E max & 2.611 & .030 & 2.551 & 2.670 & 53.394 & $.000^{*}$ \\
\hline Obsidian & 2.299 & .030 & 2.239 & 2.358 & & \\
\hline
\end{tabular}

Significance level $\mathrm{P}<0.05$, * Significant

TABLE (6C) Results of 2 ways ANOVA test for color difference

\begin{tabular}{|l|c|c|c|c|c|}
\hline Source & Type III Sum of Squares & df & Mean Square & F & Sig. \\
\hline -Etch_time & 105.144 & 3 & 35.048 & 640.808 & $.000 *$ \\
\hline -Group & 2.920 & 1 & 2.920 & 53.394 & $.000 *$ \\
\hline -Etch time * Group & 18.537 & 3 & 6.179 & 112.975 & $.000 *$ \\
\hline
\end{tabular}

Significance level $P<0.05$, *Significant

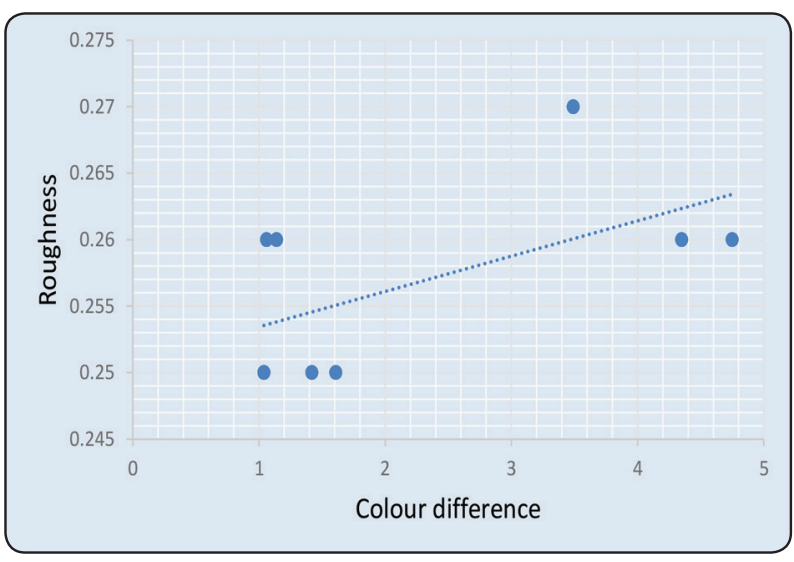

Fig. (3a) Scatter plot showing strong positive correlation between color difference and roughness in E-max group

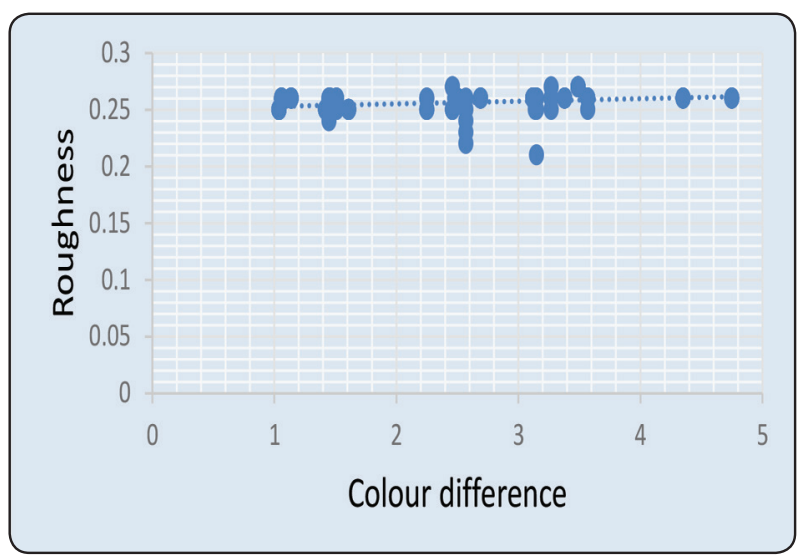

Fig. (3c) Scatter plot showing moderate positive correlation between color difference and roughness

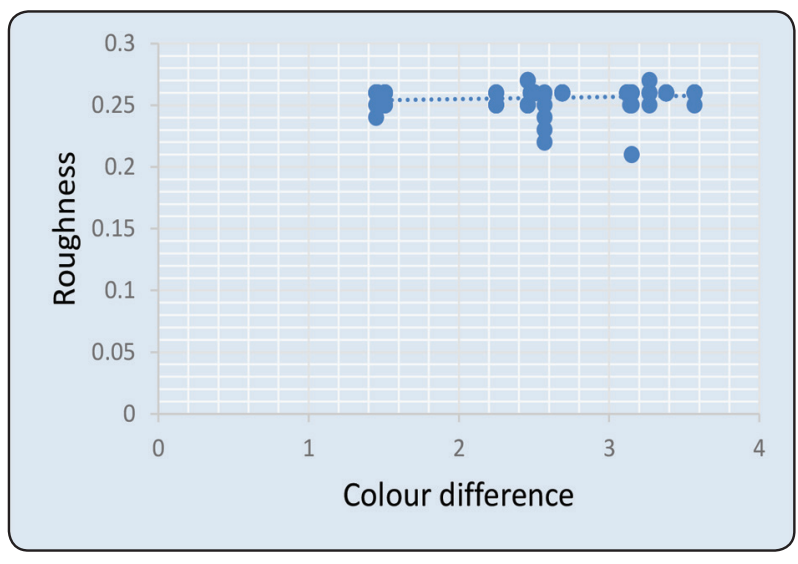

Fig. (3b) Scatter plot showing weak positive correlation between color difference and roughness in obsidian group

TABLE (7) Results of Pearson's correlation test

\begin{tabular}{llc}
\hline & & Correlation \\
\hline \multirow{2}{*}{ E max } & Pearson Correlation & .820 \\
& P value & $.000^{*}$ \\
& significance & Strong positive \\
\hline \multirow{3}{*}{ Obsidian } & Pearson Correlation & .100 \\
& P value & $.446 \mathrm{NS}$ \\
& significance & Weak positive \\
Overall (Both & Pearson Correlation & .335 \\
groups) & P value & $.000^{*}$ \\
& significance & Moderate positive \\
\hline
\end{tabular}

Significance level $P<0.05$, *Significant, NS=non-significant 


\section{ESM}

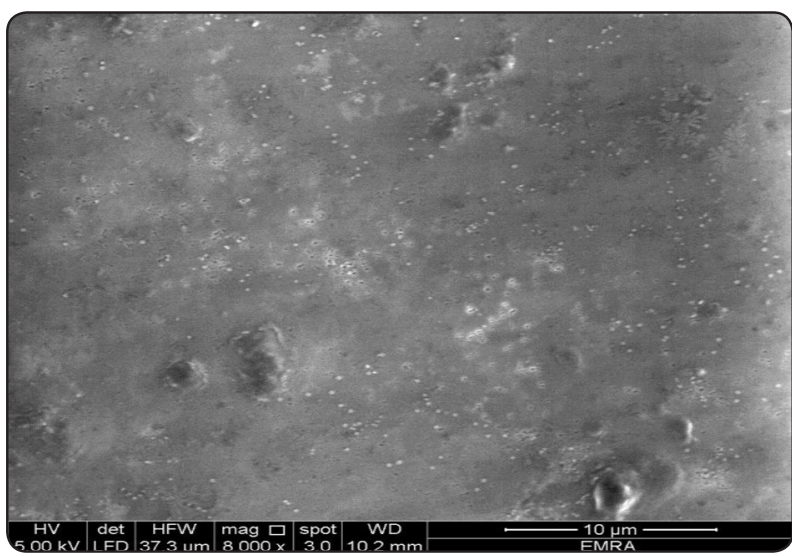

Fig. 4 (a) electron microscopic scanning of Control group (E max)

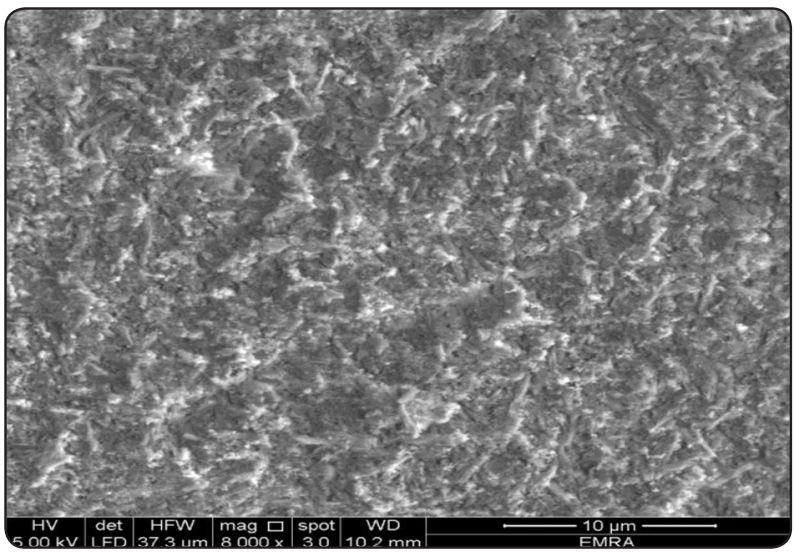

Fig. 5 (a) Electron microscopic scanning of 20 second treatment (E max)

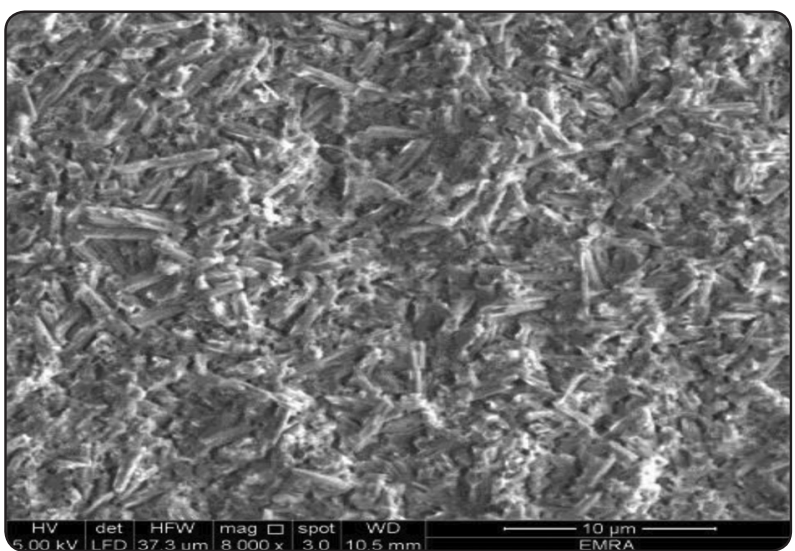

Fig. 6 (a) Electron microscopic scanning of 40 second treatment (E max)

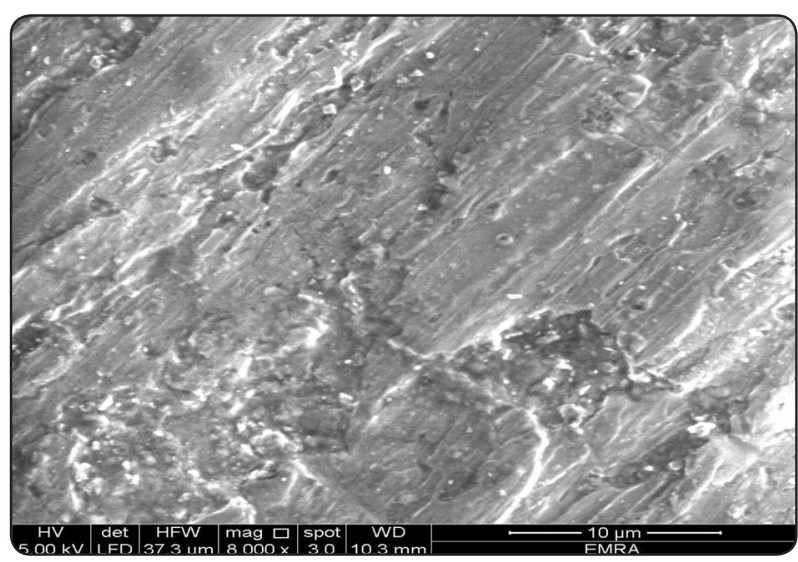

Fig. 4 (b) electron microscopic scanning of Control group (obsidian)

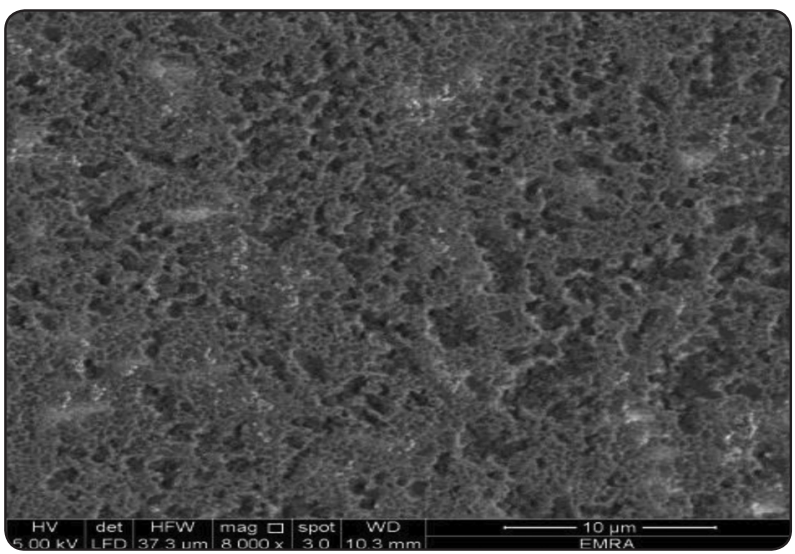

Fig. 5 (b) Electron microscopic scanning of 20 second treatment(obsidian)

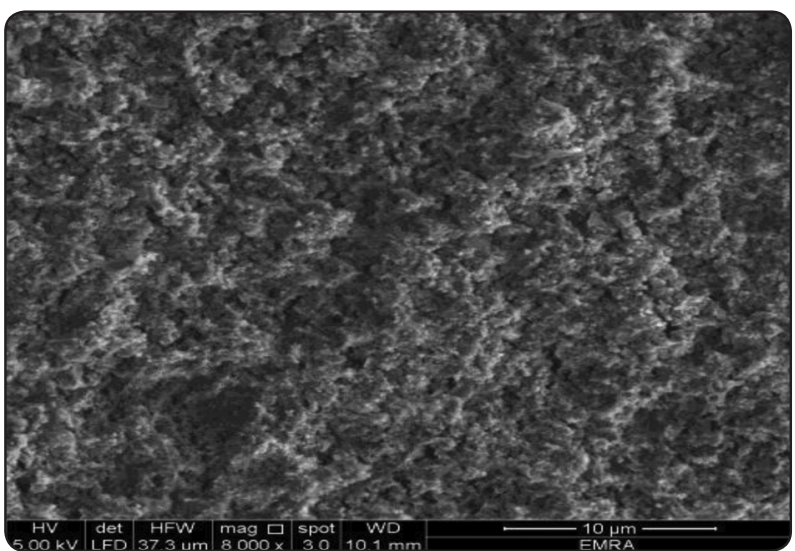

Fig. 6 (b) electron microscopic scanning of 40 second treatment (obsidian) 


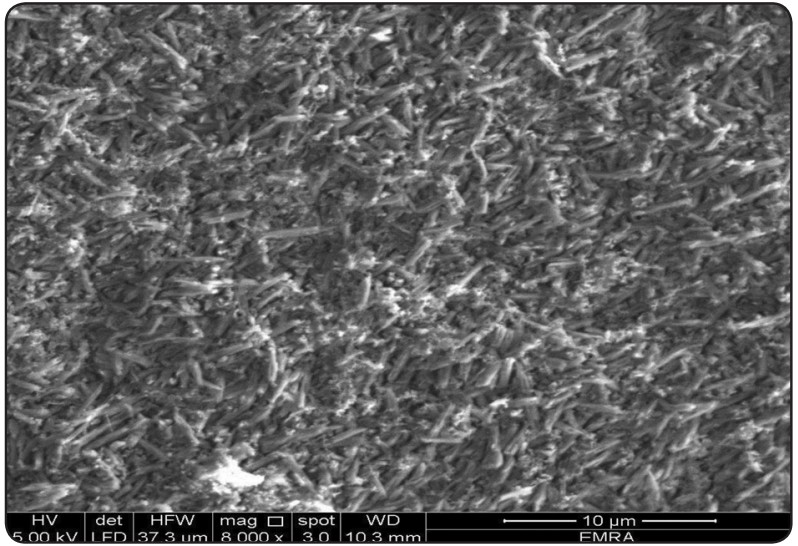

Fig. 7 (a) electron microscopic scanning of 60 second treatment (E max)

\section{DISCUSSION}

Improvements in $\mathrm{CAD} / \mathrm{CAM}$ technology have led to development of metal free restorative materials with different mechanical and optical properties. Regardless of the microstructure of the restorative material, adhesive luting protocol requires conditioning of the intaglio surface of the restoration to enhance the bond strength of restorative material to resin cement. It has been reported that surface treatment affects not only bond strength but also mechanical and optical properties of ceramics. Therefore, this in vitro study investigated the effect of different surface treatments on color of current $\mathrm{CAD} / \mathrm{CAM}$ restorative materials.

In this study; etching was done with HF acid with four different etching times $(0,20,40$, and 60). It is known that HF etching of porcelain provides the necessary surface roughness to mechanical interlocking but over etching could have a weakening effect on the porcelain. Therefore, it is important to know the adequate HF etching time for micromechanical retention without weakening the ceramic. This is the reason why the present study investigated the adequate etching protocol for a lithium disilicate-based glass ceramic.

Numerous studies have been conducted comparing the effects of different etchants on the

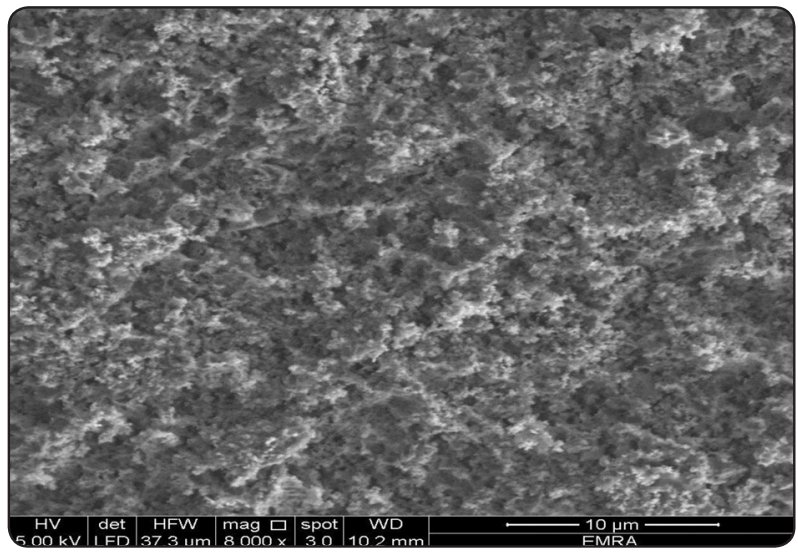

Fig. 7 (b) electron microscopic scanning of 60 second treatment (obsidian)

microstructure of glass-ceramics (18, 19, 22,23and 24). previous study found that HF produced the most aggressive etching pattern with the most prominent topographic pattern on all dental ceramics examined due to the high roughness values obtained $(\mathrm{Ra}=1.4 \mu \mathrm{m}, \mathrm{Rq}=2.1 \mu \mathrm{m}$, and $\mathrm{Rt}=39.8 \mu \mathrm{m})$ compared with acidulated phosphate fluoride.

Regarding the etching time, many studies have been done with different kinds of ceramics and HF etchants. Chen et al ${ }^{(25)}$. evaluated two HF etchants $(2.5$ and $5 \%)$ and seven different etching times $(0$, $30,60,90,120,150$, and $180 \mathrm{sec})$. Wolf et al ${ }^{(6)}$ evaluated the surface roughness of feldspathic porcelain etched with $9.5 \% \mathrm{HF}$ for $30,60,150$, and $300 \mathrm{sec}$ and the tensile bond strength to resin. The authors found a positive correlation between ceramic surface roughness and increasing $\mathrm{HF}$ etching time ${ }^{(26,27,28)}$, which agreed with other studies and with the present study.

In the current study, the hydrofluoric acid treatment of ceramic surfaces for 60 seconds showed the highest roughness value with significant difference with the other groups. This may attributed to the further loss of the glassy phase around the crystals, which exposed the crystal structure.

The emax samples showed a significant surface roughness more than the obsidian samples; this may 
attributed to the morphological difference between the two types of crystals. The lithium disilicate crystals in e max material process a needle like or tetragonal shape. While the lithium silicate crystals in obsidian material process a spherical or monoclinic shape. So that after removal of glassy matrix the needle like crystals will project sharper ${ }^{29,30)}$ and more than the other spherical lithium silicate crystals.

In case of short duration HF etching, it acts on localized areas, promoting dissolution of small amount of the glass matrix and impurities (31,32). Increasing duration leads to more homogeneous surface, due to extensive loss of the glassy matrix and pullout of lithium silicate/zirconia grains,

In the current study, the highest color difference value $\Delta \mathrm{E}$ was found in samples treated with 60 second hydrofluoric acid etching for both groups' e max as well as obsidian. Increasing itching time leads to further loss of glassy matrix ${ }^{(33)}$. So in the highest etching time (60 second) the largest amount of glassy matrix removed. which agreed with other studies $^{(34,35,36,37)}$

This glassy matrix is responsible for the translucency of ceramic. So that the maximum opacity was found in 60 second etching time group as it processes the maximum amount of crystals and the least amount of glassy matrix.

The higher mean value of color difference was in e-max groups than obsidian with statistically significant difference. This will be attributed to the higher surface roughness of e-max that leads to increasing the obesity of the material.

In the present study, SEM images of the etched and unetched ceramic surfaces noticeably represented the effect of the different etching durations on the microstructure of the glass ceramic. SEM images revealed numerous irregularities and voids in the etched ceramic surfaces as well as elongated lithium silicate crystals in comparison with the unetched ceramic surfaces, which displayed homogenous patterns. This is explained by the selective removal of the glassy matrix in the treated specimens exposing the underlying crystalline structure. In addition, as the etching periods increased, the size and number of the voids also increased as was seen in specimens etched for 60 seconds versus those etched for 10 seconds, which demonstrated fewer microstructure alterations.

\section{REFERENCES}

1- Anusavice Kj. Phillips. Science of dental materials. $11^{\text {th }}$ edition. St. Louis: Elsvier; 2003. P. 655 - 719

2- Lehner CR and Scharer P. All-Ceramic crowns. Curr oper Dent. 1992; 2: $42-62$

3- Fradeani M and Redemagni M. An 11-year clinical evaluation of leucite reinforced glass ceramic crowns : a retrospective study, Quintessence int. 2002; 33: 503 - 510

4- Wittneben JG, Robert FW, Weber HP, Gallucci GO. A systematic review of the clinical performance of CAD/ CAM single-tooth restorations. Int J Prosthodont. 2009;22: 466-71

5- Hooshmand T, Parvizi S, Keshvad A. Effect of surface acid etching on the biaxial flexural strength of two hot-pressed glass ceramics. J Prosthodont2008;17:415-9.

6- Wolf DM, Powers JM, O'Keefe KL. Bond strength of composite to etched and sandblasted porcelain. Am J Dent .1993;6:155-8.

7- Probster L, Geis J and Kirchner E. Invitro evaluation of a glass- ceramic. Restorative material. J oral Rehabil. 1997; 24: $636-645$

8- Albakry M, Guazzato $M$ and Swain Mv. Biaxial Flexural strength, elastic Moduli, and x-ray diffraction characterization of three pressable all ceramic materials. $\mathrm{J}$ prosthet Dent. 2003; 89: 374 - 380

9- Gormana CM, MC Devitta WE and Hillb RG. Comparison of two heat-pressed all -ceramic dental materials. Dent mat. 2000; 16: $389-395$

10- Apel E, Deubener J, Bernard A, Holand M, Muller R, Kapper TH, Rheinberger V and Holand W. Phenomena and mechanisms of crack propagation in glass-ceramics. $\mathrm{J}$ Biomed Mat. 2008: 313 - 325 
11- Chung H, Liao J, Duh J and Chan D. The effect of repeated heat-pressing on properties of pressable glass-ceramics: J Oral Rehabil. 2008; $10: 1365$ - 2842

12- Holand w, Schweiger M, Frank M and Rheinberger V. A comparison of microstructure and properties of the IPS Empress and the IPS Empress Glass ceramics. J Biomed Mater Res. 2000; 53: 297 - 303

13 - Wen G, Zheng X and Song L. Effects of $\mathrm{P}_{2} \mathrm{O}_{5}$ and sintering temperature on microstructure and mechanical properties of lithium disilicate glass-ceramics. Acta Materialia. 2007; $55: 3583-3591$

14- Sang - Chun Oh. Mechanical properties of reused lithium disilicate glass-ceramics of IPS Empress 2 system. J Korean Acad prosthodont. 2002; 40: 572 - 576

15- Russel C. Oriented crystallization of glass. A review. J non crystal solids. 1997; 219: $212-218$

16- Ivoclar vivadent

17- Tsujimoto, A., Barkmeier, W.W., Takamizawa, T., Wilwerding, T.M., Latta, M.A., Miyazak. Interfacial characteristics and bond durability of universal adhesive to various substrates. Oper. Dent. 2017; 42 (2), E59-E70.

18- Sattabanasuk, V., Charnchairerk, P., Punsukumtana, L., Burrow, M.F. Effects of mechanical and chemical surface treatments on the resin-glass ceramic adhesion properties. J. Investig. Clin. Dent. 2016; 1-9.

19- Sato, T.P., Anami, L.C., Melo, R.M., Valandro, L.F., Bottino, M.A. Effects of surface treatments on the bond strength between resin cement and a new zirconiareinforced lithium silicate ceramic. Oper. Dent. 2016; 41 (3), 284-292. http://dx.doi.org/10.2341/14-357-L.

20- Traini, T., Sinjari, B., Pascetta, R., Serafini, N., Perfetti, G., Trisi, P., Caputi, S., 2016. The zirconia-reinforced lithium silicate ceramic: lights and shadows of a new material. Dent. Mater. J. 35 (5), 748-755.

21- Leite, F.P.P., Özcan, M., Valandro, L.F., Moreira, C.H.C., Bottino, M.A., Kimpara, E.T. Effect of the etching duration and the ultrasonic cleaning on microtensile bond strength between feldspathic ceramic and resin cement. J. Adhes. 2013; 89, 159-173.

22- TP Sato, LC Anami, RM Melo, LF Valandro, and MA Bottino Effects of Surface Treatments on the Bond Strength Between Resin Cement and a New Zirconia-reinforced Lithium Silicate Ceramic Operative Dentistry May/June 2016, Vol. 41, No. 3, pp. 284-292
23- Hatem M.El-Damanhoury ${ }^{\mathrm{ab} M a r i a}$ D.Gaintantzopoulou ${ }^{\mathrm{c}}$ Self-etching ceramic primer versus hydrofluoric acid etching: Etching efficacy and bonding performance. Journal of Prosthodontic Research , January 2018, Pages 75-8

24- J Puppin-Rontani, D Sundfeld, AR Costa, AB Correr, RM Puppin-Rontani, GA Borges, MAC Sinhoreti, and L Correr-Sobrinho (2017). Effect of Hydrofluoric Acid Concentration and Etching Time on Bond Strength to Lithium Disilicate Glass Ceramic. Operative Dentistry: November/December 2017, Vol. 42, No. 6, pp. 606-615.

25- Chen JH, Matsumura H, Atsuta M. Effect of different etching periods on the bond strength of a composite resin to a machinable porcelain. J Dent 1998;26:53-58.

26- Lucas Hian da SILVA(a) Erick de LIMA(b) Ranulfo Benedito de Paula MIRANDA(b) Stéphanie Soares FAVERO(b) Ulrich LOHBAUER(c) Paulo Francisco CESAR(b). Dental ceramics: a review of new materials and processing methods Critical Review.Dental Materials/ Dentistry May 25, 2017133147 -

27- Venturini, A.B., Prochnow, C., May, L.G., Bottino, M.C., Felipe Valandro, L., 2015a. Influence of hydrofluoric acid concentration on the flexural strength of a feldspathic ceramic. J. Mech. Behav. Biomed. Mater. 48, 241-248. 2015.03 .025

28- Çağrı Ural1, İbrahim Duran1, Betül Evmek1, İdris Kavut1, Seda Cengiz and Emir Yuzbasioglu3,4. Light transmittance and surface roughness of a feldspathic ceramic CAD-CAM material as a function of different surface treatments. Ural et al. BMC Oral Health (2017) 17:16

29- Jaiane Bandoli Monteiroa, Marcelo Gallo Oliania, Luis Felipe Guilardib, Catina Prochnowb Fatigue failure load of zirconia-reinforced lithium silicate glass ceramic cemented to a dentin analogue. J Prosthet Dent77 (2018) 362-372

30- Gabriel Kalil Rocha Pereirab,c, Marco Antonio Bottinoa, Renata Marques de Meloa, Luiz Felipe Valandrob. Effect of etching time and hydrofluoric acid concentration, Journal of the Mechanical Behavior of Biomedical Materials 77 (2018) 375-382

31- Luo Xiaoping, Ren Dongfeng, Nick Silikas Effect of etching time and resin bond on the flexural strength of IPS e.max Press glass ceramic J Prosthet Dent December 2014

32- Suk-Ho Kang 1Juhea Chang 2, Ho-Hyun Son Flexural strength and microstructure of two lithium Disilicate glass ceramics for CAD/CAM restoration in the dental clinic Operative Dentistry 134-140 
33- Vinícius Esteves Salgado, Guilherme Ferreira Rego, Luis Felipe Schneider, Rafael Ratto de Moraes, Larissa Maria Cavalcante. Does translucency influence cure efficiency and color stability of resin-based composites?Dental Materials, Vol. 34, Issue 7

34- Abdulaziz Al Ben Ali BDS, MSc, FACP Kiho Kang DDS, DMD, MS Matthew D. Finkelman PhD Roya Zandparsa DDS, MSc, DMD Hiroshi Hirayama DDS, DMD, MS The Effect of Variations in Translucency and Background on Color Differences in CAD/CAM Lithium Disilicate Glass Ceramics First published: 26 July 2013 J Prosthet Dent Volume 23, Issue 3

35- Fu Wang, DDS, PhD Hidekazu Takahashi, DDS, PhD Hidekazu Takahashi Naohiko Iwasaki, RTD, PhD. Trans- lucency of dental ceramics with different thicknesses. J Prosthet Dent. July 2013Volume 110, Issue 1, Pages 14-20

36- Alessandro Vichi DDS, MS, PhD Michele Carrabba DDS Rade Paravina DDS, MS, PhD Marco Ferrari MD, DDS, PhD Translucency of Ceramic Materials for CEREC CAD/ CAM System J ethetic and restorative dentistry 29 June 2014 Volume26, Issue4Pages 224-231

37- Gülcan Coşkun Akar, DDS, PhD, Gürel Pekkan, DDS, PhDPhD Gürel Pekkan, Gürel Pekkan, Ebru Çal, Gürcan Eskitaşçıŏlu, Mutlu Özcan Effects of surface-finishing protocols on the roughness, color change, and translucency of different ceramic systems DrMedDent, PhD August 2014Volume 112, Issue 2,Pages 314-321 J Prosthet Dent. 